WACQUANT, Loïc "La futilidad y necesidad de los derechos humanos en la era de hiper inflación carcelaria".

Polít. crim. Vol. 12, No 24 (Diciembre 2017), Doc. 2, pp. 1264-1274.

[http://www.politicacriminal.cl/Vol_12/n_24/Vol12N24D2.pdf]

\title{
La futilidad y necesidad de los derechos humanos en la era de hiper inflación carcelaria
}

\section{The futility and necessity of human rights in an era of penitentiary hiper inflation}

\author{
Loïc Wacquant \\ 1oic@berkeley.edu \\ University of California, Berkeley* \\ Centre européen de sociologie et de science politique, París
}

A través del transcurso de doce años, he tenido la oportunidad y el privilegio de compartir mi investigación sobre el nexo entre encarcelamiento y marginalidad urbana con un amplio rango de personas que no provienen del mundo académico. He hablado y debatido con líderes y el personal de los servicios de prisiones en Francia, Holanda, Brasil y Argentina. También he conversado con oficiales y profesionales interesados sobre las tendencias y políticas del sistema de justicia criminal en las Naciones Unidades, la OCDE, el parlamento italiano, junto con tener nociones de gobernaciones regionales y de ciudades (que van desde el distrito de Seine-Saint-Denis al noreste de Paris, y de la Generalitat de Catalunya a ciudades muy lejanas como Rotterdam, Roma y Río de Janeiro). Doy la bienvenida a esta oportunidad para dialogar y aprender mutuamente en este día en Santiago con Gendarmería de Chile.

\section{La prisión en la práctica profesional y la teoría social}

En primer lugar, me gustaría dejar en claro que yo no estoy aquí para inculpar o disculpar a Gendarmería por medio de mis palabras o actos, no porque yo sea tímido o aprensivo, sino simplemente porque los sociólogos no son moralistas. No estamos en el asunto para juzgar instituciones, decidiendo quien es "culpable" y quien es "inocente", asignando culpas por condiciones sociales indeseadas y por resultados humanos no deseados. Estamos en la cuestión para analizar operaciones ordinarias, contradicciones incorporadas, los efectos

\footnotetext{
${ }^{*}$ Loïc Wacquant es profesor de sociología en la Universidad de California, Berkeley, e investigador del Centro de Sociología Europea, Paris. Sus intereses incluyen la marginalidad urbana, penalidad, sociología del cuerpo, y teoría social. Sus libros han sido traducidos en dos docenas de idiomas, que incluyen en el idioma castellano: Las Cárceles de la miseria (2000, con una versión expandida el 2010); Entre las cuerdas. Cuadernos etnográficos de un aprendiz de boxeador (2004); Los Condenados de la ciudad. Gueto, periferias, Estado (2007) y Castigar a los pobres. El gobierno neoliberal de la inseguridad social (2010).

Esta es una versión revisada de los apuntes preparados para un discurso de apertura enviado a Gendarmería de Chile, División de Derechos Humanos, el 16 de octubre del año 2013 en el hotel Gran Palace, Santiago de Chile. Le agradezco a Gendarmería de Chile por esta invitación. También le agradezco a Alejandro Arévalo, que encabeza la Unidad de Protección y Promoción de Derechos Humanos por incluirme en este debate; a Guillermo Sanhueza por su incansable trabajo detrás de la escena; y a Megan Comfort por sus astutas observaciones durante y después de nuestra visita a la prisión La Penitenciaría. Traducción realizada por Sebastián Galleguillos Agurto.
} 
WACQUANT, Loïc "La futilidad y necesidad de los derechos humanos en la era de hiper inflación carcelaria”.

ramificadores de las instituciones, las funciones sociales que cumplen y las ficciones sociales que sostienen ${ }^{1}$. Quiero enfatizar esto al comienzo para disipar la visión errónea sobre el rol que cumplen los sociólogos, predominante entre la ciudadanía educada, así como entre muchos de mis colegas (quienes piensan que poniendo el adjetivo de "crítica" antes de sociología los hace desperdiciar tinta y papel).

Segundo, no estoy aquí para dar lecciones a Gendarmería de Chile: los académicos debiesen siempre ser cuidadosos cuando se dirigen al mundo profesional, ya que ellos residen la mayoría del tiempo en un mundo abstracto de textos, por encima y protegido de la urgencia de tareas prácticas. En contraste, los profesionales tienes sus manos sucias al resolver cosas en cualquier campo que operen. No tengo experiencia haciendo funcionar una prisión, pero sí tengo suficiente idea de las complejidades que involucra para saber que estoy mal posicionado para instruir a cualquiera que sabe cómo administrar su propio establecimiento. Preferiría no contar mi larga visita durante la mañana en la Penitenciaría, sin embargo, tan informativa ha sido, que la aprovecharé de base para algún consejo. Las visitas a prisiones son interesantes para percibir que es lo que quieren exhibir los guardias a los outsiders; ellas nos dicen mucho acerca de la visión que los oficiales quieren proyectar, más que sobre la realidad diaria bajo llave.

Pero aquí está la ventaja de estar alejado de una institución determinada; puedes estudiarla, analizarla, descomponerla en sus elementos constitutivos, y aprender de la comparación de sus unidades, épocas y límites geográficos. La contribución que el sociólogo puede hacer en torno a la discusión sobre derechos humanos tras la rejas es entregar un punto de vista amplio desde fuera; entregar las lecciones de las experiencias históricas e internacionales para influir en los debates contemporáneos en un determinado país; proporcionar un marco analítico que puede hacer posible retroceder de la urgencia de la práctica y del fuego del debate político, reformular preguntas, y señalar algunas consecuencias imprevistas y contradicciones de la privación penal, así como también vincular la prisión a otras instituciones más allá de sus muros.

Diferentes tradiciones teóricas dentro de las ciencias sociales articulan diferentes conexiones entre el castigo e instituciones no judiciales. A grandes rasgos, podemos distinguir cinco hilos teóricos y vínculos organizacionales:

1) Clase y economía: la tradición neo-marxista describe a la justicia criminal como un instrumento de control material cuyo objetivo principal son los pobres, y cuyas tendencias reflejan las esferas de producción, particularmente la condición de obreros. Su tópico común es la macro correlación entre encarcelamiento y desempleo. Punishment and social structure de Rusche y Kirschheimer es generalmente citado como el texto canónico de esta tradición- erradamente desde mi punto de vista- desde que presenta un modelo multifactorial tomando en

\footnotetext{
${ }^{1}$ Max Weber lo escribió mejor en sus ensayos sobre The Methodology of the Social Sciences: nuestra tarea es "el ordenamiento analítico de la realidad empírica", el cual es diferente de evaluar sus valores estéticos, morales o tenor político. Al respecto, véase WEBER, Max, The Methodology of the Social Sciences, Glencoe: Free Press, 1948.
} 
Polít. crim. Vol. 12, No 24 (Diciembre 2017), Doc. 2, pp. 1264-1274.

[http://www.politicacriminal.cl/Vol_12/n_24/Vol12N24D2.pdf]

consideración variables extra económicas como la capacidad física, religión y sexualidad, lo que lo coloca más cerca de Weber que de Marx.

2) Comunidad y moralidad: la tradición descendiente de Émile Durkheim y de la escuela francesa de sociología construye el castigo como un lenguaje, un medio de comunicación simbólica que captura las emociones colectivas para destacar y exaltar normas compartidas. El objetivo aquí no es el criminal sino el respeto ciudadano de la ley, siendo un tópico favorito el espanto del crimen y las ceremonias judiciales que encarnan el tenor apasionado del castigo y que sirven para trazar los límites entre insiders y outsiders. Un estudio paradigmático es el de Kai Erikson, que analiza los juicios de brujos en Salem durante el siglo diecisiete de la colonia puritana de Massachussets en Wayward Puritans.

3) Profesionales de la racionalidad, mentalidades y sensibilidades culturales de elite: una tercera línea de trabajos que toma lugar después del análisis clásico de ley, burocratización y racionalización por Max Weber y su epígono Norbert Elias. Esto releva la aparición de los oficiales especialistas en el manejo del crimen, el cambio de actitudes de las elites hacia el cuerpo, pena, y desviación, y como estas han formado gradualmente el castigo "civilizado". El historiador holandés Pieter Spierenburg ofrece una clásica posición en su libro The Spectacle of Suffering que traza la desaparición del espectáculo público de sufrimiento físico desde el siglo XVI al siglo XIX en Europa. Ubico a Foucault dentro de esta extensa tradición neowebeariana, dada su preocupación por la singularidad de occidente y la racionalización de facto de la dominación a través de difusión social de las "disciplinas" diseñadas para hacer a los cuerpos "dóciles y útiles" en Discipline and Punish.

4) Forjando la desviación criminal, identidad penal y el yo: Foucault también ofrece un link a la tradición microsociológica, cuyas raíces se encuentran en la escuela norteamericana del interaccionismo simbólico, preocupada por los mecanismos sociales que producen desviación, dan forma a la identidad y moldean la personalidad del criminal y del interno. Los libros Asylums de Erving Goffman y The Felon de John Irwin son posiciones ejemplares de esta aproximación que nos entrega una comprensión del castigo desde el punto de vista del castigado y sus estrategias de afrontamiento.

5) El estado como poder simbólico y campo burocrático: el último y más reciente teórico que escribió en el tema es mi mentor y amigo, el sociólogo francés Pierre Bourdieu. Bourdieu nos entrega dos conceptos para repensar el castigo: la noción de "poder simbólico" apunta que la monopolización sobre la capacidad de imponer clasificaciones en sociedad y de dañar el honor de sus miembros es una forma de sanción penal; la noción de "campo burocrático" sugiere que hay una lucha dentro del estado entre agencias rivales que intentan resolver problemas sociales aplicando un tratamiento de apoyo social ( la mano femenina izquierda) o sanciones disciplinarias ( la mano derecha masculina). En mi libro Castigar a los pobres (2009), adapto estas dos nociones para demostrar que el resurgimiento de la prisión 
WACQUANT, Loïc "La futilidad y necesidad de los derechos humanos en la era de hiper inflación carcelaria”.

dentro de los pasados treinta años forma parte de la construcción del estado neoliberal y su política de penalización de la pobreza.

Esta breve descripción tiene el propósito de apuntar que, lejos de ser una institución aislada, la prisión se encuentra simultáneamente vinculada a una multiplicidad de instituciones sociales y fuerzas, ellas mismas interconectadas: la economía, emociones cívicas, sensibilidades de la elite y formas burocráticas, las identidades elaboradas por el castigador y el castigado, y las luchas modeladas dentro del estado sobre cómo definir y distribuir los bienes públicos, incluyendo el bien negativo del castigo criminal.

Aquí quiero destacar esta última variable: la noción de derechos humanos es típicamente invocada por elites educadas con una apuesta por la expansión del rol de la mano izquierda estatal, en oposición a sus contrapartes de la mano derecha que invocan la racionalidad económica y necesidades presupuestarias para minar la extensión y aplicación de los costosos derechos. El ministro de justicia, desde donde se supervisa el sistema de prisiones, se encuentra atravesado por la misma tensión entre el polo izquierdo comprometido con derechos y rehabilitación, y un polo derecho cegado por la retribución y la implementación rígida de la ley y el orden como asunto de la autoridad estatal. Yo sospecho que en este auditorio hay miembros de Gendarmería de Chile que están comprometidos con la defensa y expansión de los derechos humanos detrás de las rejas, y otros que ven los mismos derechos humanos como inútiles lujos e irresponsables distracciones de su misión, si es que no lo ven como un Caballo de Troja de una revolución izquierdista. El hecho de que yo esté invitado el día de hoy a dar este discurso, sumado a la mirada severa de atención militar de mi audiencia, es indiciario de que la lucha entre ambos polos es incierta y asimétrica ${ }^{2}$.

La aproximación de Bourdieu nos invita a realizar tres pasos que son útiles para contextualizar el debate sobre los derechos humanos en prisión ${ }^{3}$. El primer paso es separar crimen de castigo y reconocer que, desde los tiempos históricos de su invención a fines del siglo XVI, la prisión penal siempre ha servido para llevar a cabo la gestión de la marginalidad urbana, y para afirmar las prerrogativas del gobernante a través del espectáculo de la sanción penal.

El siguiente paso es tratar la política del estado social de bienestar y la política penal como dos caras de la misma moneda de la política de pobreza, reconociendo que estas dos corrientes políticas deben entenderse juntas, en la medida que ellas tratan a la misma población y despliegan los mismos tropos discursivos y técnicas administrativas. El tercer paso es mantener unidos los momentos materiales y simbólicos del castigo, para dar cuenta

\footnotetext{
${ }^{2}$ Me gustaría hipotetizar además que los defensores de los derechos humanos dentro del espacio social polarizado dentro de Gendarmería de chile son tendientes a ser más jóvenes, más femeninos, dotados con credenciales universitarias y trayectorias que han comenzado afuera de la aplicación de la ley, mientras que sus detractores ostentan las propiedades opuestas. Sobre el fondo del asunto, véase el completo estudio de la reciente transformación de las instituciones penales y políticas en Chile en HATHAZY, Paul Carlos, Democratizing Leviathan: Bureaucrats, Experts and Politics in the Transformation of the Penal State in Argentina and Chile. Berkeley, unpublished Ph.D dissertation, pp. 1-332, 2013.

${ }^{3}$ Al respecto, WACQUANT, Loïc, "The Wedding of Workfare and Prisonfare Revisited", Social Justice 38, 1-2 (Spring, 2011), pp. 203-221.
} 
Polít. crim. Vol. 12, No 24 (Diciembre 2017), Doc. 2, pp. 1264-1274.

[http://www.politicacriminal.cl/Vol_12/n_24/Vol12N24D2.pdf]

que las funciones de control y comunicación, asociadas con las escuelas marxistas y durkheimianas respectivamente, no son antinómicas sino complementarias e incluso mutuamente sostenidas. Cuando arrestas a un sospechoso y encarcelas a un condenado, estas simultáneamente ejerciendo fuerza física y simbólica: estas restringiendo tanto los derechos de esa persona como también transmitiendo un mensaje moral mediante la amputación de su calidad del miembro en el pacto cívico y ensuciando su dignidad.

\section{Conectando los derechos humanos por dentro y los derechos sociales por fuera de la cárcel}

La pregunta por los derechos humanos es ante todo una pregunta jurídica y moral, no una pregunta sociológica, por lo que me siento mal posicionado para abordarla. Estoy consciente de que existe un extenso y dinámico dominio de la investigación en la intersección entre ley, sociología política, y estudios de paz que despliegan esta noción ${ }^{4}$. Desde mi punto de vista, sin embargo, en el actual contexto de hiperinflación carcelaria y reforzamiento del estado penal a lo largo de América Latina ${ }^{5}$, los derechos humanos forman lo que Kenneth Burke llama pantalla terminística ${ }^{6}$, un discurso que encubre la realidad de las catastróficas condiciones tras las detenciones masivas- evocadoras de las prisiones en las mazmorras medievales ${ }^{7}$-conformando un conjunto de categorías formales que ofuscan los intereses de quienes lo invocan ${ }^{8}$.

Me gustaría dar vuelta la pregunta y cuestionar: ¿Cuándo los derechos humanos son invocados tras las rejas? Respuesta: cuando los derechos económicos y sociales fundamentales son diariamente violados tras las rejas, como es el caso de Latinoamérica producto de la combinación de extrema inequidad social, extendida pobreza, y la realidad política del descuido de la ley ${ }^{9}$. A riesgo de parecer paradójico por sí mismo, argumentaré

\footnotetext{
${ }^{4}$ SOMERS, Margaret R., ROBERTS, Cristopher N.J, “Toward a New Sociology of Rights: A Genealogy of 'Buried Bodies' of Citizenship and Human Rights”, Annual Review of Law and Social Science 4, (2008), pp. 385-425; CLAPHAM, Andrew, Human Rights: A Very Short Introduction, Oxford: Oxford University Press, 2016.

${ }^{5}$ MULLER, Markus-Michael, "The Rise of the Penal State in Latin America”, Contemporary Justice Review 15, no. 1 (2012), pp- 57-76; HATHAZY, Paul; MULLER, Markus-Michael, "The Rebirth of the Prison in Latin America: Determinants, Regimes and Social Effects.”, Crime, Law and Social Change 65, no 3 (2016), pp. 113-135.

${ }^{6}$ BURKE, Kenneth, The Philosophy of Literary Form, Berkeley: University of California Press, 1957.

${ }^{7}$ GELTNER, Guy, The Medieval Prison: A Social History, Princeton, NJ: Princeton University Press, 2008.

${ }^{8}$ Sobre este punto, recomiendo la lectura, GUILHOT, Nicolas, The Democracy Makers: Human Rights and International Order, New York: Columbia University Press, 2005, el cual muestra como el movimiento transnacional por la democracia y los derechos humanos ha sido secuestrado por expertos y convertido en un instrumento de reforma neoliberal. Para miradas disidentes que documentan una perspectiva más positiva del rol de los derechos humanos, véase para Europa DIRK, van Zyl Smit; SNACKEN, Sonja, Principles of European Prison Law and Policy: Penology and Human Rights, Oxford: Oxford University Press, 2009; SIKKINK, Kathryn, Mixed Signals: US Human Rights Policy and Latin America, Ithaca, NY: Cornell University Press, 2007; y para Latinoamérica CARDENAS, Sonia, Human Rights in Latin America: A Politics of Terror and Hope, Philadelphia, University of Pennsylvania Press, 2011.

${ }^{9}$ HOFFMAN, Kelly; CENTENO, Miguel Angel, "The Lopsided Continent: Inequality in Latin America", Annual Review of Sociology 29, (2003), pp. 363-390; MÉNDEZ, Juan E.; O'DONNELL, Guillermo A.; PINHEIRO, Paulo Sérgio (Eds.), The (Un) Rule of Law and the Underprivileged in Latin America. South Bend, Indiana: University of Notre Dame Press, 1999.
} 
WACQUANT, Loïc "La futilidad y necesidad de los derechos humanos en la era de hiper inflación carcelaria”.

que hablar de derechos humanos en las cárceles de sociedades que continuamente violan los estándares básicos de la dignidad humana al fondo de su estructura de clase es a la vez fútil e indispensable.

El hablar de derechos humanos detrás de las rejas es un ejercicio fútil por dos razones. La primera es que la prisión es en sí fundamentalmente una institución fuera de la ley: funciona exlex, fuera de la ley. Bajo la presión de la necesidad, las autoridades correccionales de todos lados violan las reglas legales que ellos supuestamente defienden. Prueba de ello es la difundida brutalidad y shockeantes penurias materiales que aquejan a los reclusos a lo largo de Latinoamérica. Incluso en países desarrollados, los códigos penitenciarios legales son poco desarrollados, débiles y solipsistas, incapaces de asignar efectivamente a los prisioneros un conjunto básico de derechos. Esto se debe a que el confinamiento es diseñado por ciudadanos zombies, existiendo en las sombras de la ley. La segunda razón es que la prisión es una institución intensamente dañosa: es un lugar de concentración de violencia y vicio; ultraja la personalidad, distorsiona la sociabilidad, y erosiona el bienestar físico y mental de quienes están allí. Es más, parece absurdo otorgar y hacer valer los derechos humanos tras las rejas cuando estos mismos derechos son negados debido a la debilidad de los derechos económicos y sociales de los ciudadanos que requieren de condiciones mínimas de actualización.

Con todo, todavía hay seguramente una utilidad para los derechos humanos: el discurso legal puede servir como guía y estímulo para mejorar los fondos y funcionamiento correccional, como arma para frenar la ilegalidad penal y para minimizar el daño humano dentro de las instalaciones carcelarias. Por esta razón, debemos invocarlos, insertarlos dentro de estándares correccionales, y buscar hacerlos cumplir mientras no encontramos nada mejor, mientras en dicho tiempo nos abocamos a una reflexión política fundamental sobre los propósitos de la encarcelación, o la falta de argumentos para sostenerla ${ }^{10}$. Pero debemos hacer esto sin ilusiones sobre sus límites, y por medio de la conexión constante derechos humanos desde dentro y derechos sociales por fuera de la cárcel.

Entonces, ¿Como podemos aplicar los derechos humanos para minimizar el vínculo entre ilegalidad y la destructividad social de la prisión? Yo propongo que sigamos tres principios. Primero, minimizar las huellas de la institución carcelaria maximizando vías alternativas de manejo de marginalidad y crimen, incluyendo tratamientos sociales, médicos y económicos sobre condiciones y conductas problemáticas, todas los cuales se focalizan en la preclusión y profilaxis en vez de la reacción punitiva ${ }^{11}$.

En segundo lugar, volver al vínculo entre inside y outside, ya que la prisión no es un espacio cerrado ni hermético. Contrario a la visión oficial, sus paredes son doblemente

${ }^{10}$ David Garland sostiene que no realmente no sabemos porque encarcelamos más debido a la "crisis del sistema penal moderno". Al respecto, GARLAND, David, "Penal Modernism and Postmodernism", en: BLOMBERG, Thomas G.; COHEN, Stanley (Eds.), Punishment and Social Control. New York: Aldine de Gruyter, 2nd expanded edition, 2003.

${ }^{11}$ Discuto estas alternativas en Wacquant: "How to Escape the Law-and-Order snare", en: WACQUANT, Loï, Punishing the Poor: The Neoliberal Government of Social Insecurity, Durham and London: Duke University Press, 2009. 
porosas, lo que significa que fuerzas externas se filtran y afectan sus operaciones y resultados (concretamente, piensen en los proveedores de servicio y visitantes que cruzan los perímetros diariamente y el contrabando de drogas, celulares, y bienes indispensables como comida, medicina, y artículos de aseo personal) y que, por otro lado, la prisión afecta profundamente los vecindarios de la relegación que son sus principales alimentadores. Esto es verdad en todos lados, pero en ningún lugar es tan cierto como en Latinoamérica, donde debido a la falta de fondos suficientes, las prisiones confían en el constante progreso trans muros para sus operaciones básicas y mantenimiento del orden de la rutina. En tercer lugar, es necesario tomar en consideración el impacto perjudicial a lo largo de la encarcelación dentro y a través de generaciones: el encierro deforma las trayectorias de vida no solo de los presos sino también de sus familias, desde sus esposas y padres a sus hijos, nietos, y a muchos de su parentesco y amigos entre medio ${ }^{12}$. La prisión es una institución que perpetúa la pobreza e induce al crimen, dentro y más allá de sus murallas.

Algunos de ustedes podrían reclamar que estos principios son demasiado amplios y genéricos, y que ellos fallan en proveer acciones inmediatas y concretas, más allá de las indispensables metas de reducir el maltrato al personal, violencia carcelaria, y mejorando la visita y acceso a programas ${ }^{13}$. Déjenme terminar haciendo unas pocas recomendaciones prácticas, pero políticamente improbables a Gendarmería de Chile y, más allá de eso, al servicio de prisiones de varios países latinoamericanos que pueden encontrar inspiración en las reformas chilenas, que pueden hacer de este país un pionero continental para la descarcelación después de ser un líder en encarcelación por tanto tiempo.

1. Reducir la grotesca sobrepoblación: los derechos humanos son una broma mientras los presos sean comprimidos como sardinas muertas. De acuerdo a los datos oficiales, la ocupación a nivel nacional de la custodia en Chile establece que es de $118 \%$, alcanzando un $130 \%$ en la Penitenciaria. Pero esto es una completa farsa: estas cifras son un sinsentido debido a que son obtenidas atestando seis hombres en celdas pequeñas que debiesen habitar a lo más dos personas. Por lo tanto, el actual nivel de sobrepoblación es cercano al 330\%. La reducción de la sobrepoblación se puede obtener reduciendo la extensión de las sentencias en todos los ámbitos por buen comportamiento (día a día), incrementando las liberaciones tempranas y expandiendo los programas de desviación (para minimizar las entradas con sentencias de corto término).

2. Establecer como objetivo una baja población penal y un límite: lanzar una política activa de descarcelamiento con metas ambiciosas. Chile debiese colocar como objetivo volver a la media de 100 presos por 100.000 habitantes, similar a la media reportada en 1981 y cercana a la media de Europa occidental. Partiendo de

\footnotetext{
${ }_{12}$ Véase el trabajo COMFORT, Megan, Doing Time Together: Love and Family in the Shadow of the Prison, Chicago: University of Chicago Press, 2008, sobre novias y esposas de convictos; BLACK, Timothy, When a Heart Turns Rock Solid: The Lives of Three Puerto Rican Brothers On and Off the Streets, New York: Vintage, 2010 para el caso de los hermanos; y WAKEFIELD, Sara; WILDEMAN, Christopher, Children of the Prison Boom: Mass Incarceration and the Future of American Inequality. New York: Oxford University Press, 2013, para el impacto multidimensional en los hijos de presos.

${ }^{13}$ SANHUEZA, Guillermo; BRANDER, Francisca; FUENZALIDA, Fernando, "First Survey on Prison Life in Chile: A Social Work Call for Prison Reform.”, International Social Work: in press, 2017.
} 
WACQUANT, Loïc "La futilidad y necesidad de los derechos humanos en la era de hiper inflación carcelaria”.

una media de 300, esto puede ser logrado recortando cerca de 12 puntos por año durante 15 años, que representa una reducción de cerca de 1.700 presos anualmente. Este promedio de 100 puede ser tratado como un límite absoluto, incentivando a las cortes para encontrar alternativas creativas y efectivas al encarcelamiento siempre y cuando esté al alcance.

3. Mover la prisión desde el ministerio de justicia al ministerio de desarrollo social (donde pertenece) y entregar programas efectivos en salud (incluyendo salud mental), asesoramiento matrimonial y familiar, formación profesional $\mathrm{y}$ empleabilidad bajo lo que yo llamo la regla del dos por uno: dos unidades de cualquier servicio serán proveídos en los distritos desposeídos, vinculados simbióticamente a la prisión por cada unidad entregada dentro de la prisión. Por ejemplo, ofrecer trabajos de construcción dentro y fuera de la cárcel de acuerdo a esta proporción. Impulsando los derechos económicos y sociales en ambos lados de las murallas de la prisión, simultáneamente cambiarán las políticas de resentimiento (que retratan falsamente la provisión de servicios correccionales como un juego de suma cero que gasta recursos de los respetuosos de la ley), y ayuda a crear un apoyo popular para este tipo de programas.

4. Reducir la shockeante miseria de la planta física de las penitenciarías como parte de un programa de vivienda pública a gran escala. Cerrar las instalaciones antiguas no aptas para el hábitat humano y remplazarlas por nuevas construcciones como parte de una política de construcción dual: por cada peso invertido en la construcción de una nueva prisión, invertir dos pesos en el mejoramiento del parque de viviendas e infraestructura en el vecindario de la relegación que son los principales alimentadores de las casas de confinamiento. En efecto, tratar las prisiones por lo que son en todos lados: un componente central de bajos ingresos y vivienda social.

5. Integrar los servicios públicos detrás de las rejas como educación, cuidados de salud y trabajo social con servicios públicos regulares desde fuera: crear un "servicio nacional" obligatorio para profesores, enfermeros, doctores, psiquiatras y asesores familiares para servir dos años dentro de las prisiones en la nómina del gobierno, un año después de obtener el título y otro año una década después. Este servicio mejorará drásticamente las condiciones de los presos, y dejará la preocupación de los profesionales del exterior sobre qué ocurre con los convictos. Proporcionar créditos de matrícula para estudiantes universitarios que enseñan educación secundaria dentro de las prisiones. Hacer que la alfabetización universal sea obligatoria: nadie debiese salir de prisión sin saber cómo leer y escribir.

6. Elevar el status y calidad del personal de prisiones: incrementar la línea de base de las calificaciones, los entrenamientos periódicos, y el pago al personal de las prisiones para posicionar al oficial correccional como un trabajo valorizado y atractivo. Enseñarles nociones básicas aplicadas en sociología, psicología y trabajo social en vez de entrenarlos solo en técnicas de mantención del orden. Esto valorizará su trabajo como también lo armonizará con los derechos tras las rejas. En particular, instruirlos en que el respeto a ley y el tratamiento justo de cada preso en 
Polít. crim. Vol. 12, No 24 (Diciembre 2017), Doc. 2, pp. 1264-1274.

[http://www.politicacriminal.cl/Vol_12/n_24/Vol12N24D2.pdf]

toda circunstancia es el fundamento de su trabajo (sabemos que no es el miedo, sino la justicia procesal la razón por la que las personas obedecen la ley) ${ }^{14}$.

7. Triplicar el presupuesto correccional en quince años, y hacer la prisión gradualmente más costosa, para que los políticos con mentalidad presupuestaria busquen alternativas de solución al encarcelamiento. Las proposiciones 3 a 6 requieren un significativo incremento en los fondos públicos. Junto con las proposiciones 1 y 2, ellas incrementarán el costo per cápita de encarcelamiento de los ofensores, que incorporará un incentivo para no sobrepasar el límite de la cárcel.

8. Circular a una campaña de información pública sobre la falla de actuales políticas penales dejando en claro a los ciudadanos que la prisión es una institución en banca rota, un instrumento contundente y brutal que no resuelve el problema del delito sino que simplemente lo esconde y desplaza; la encarcelación no reduce la reincidencia y expande la habilidad de los condenados para llevar una vida lícita y productiva una vez que ellos retornan a la sociedad ( lo que casi todos ellos hacen); el encarcelamiento es enormemente más costoso que las respuestas alternativas, y se está volviendo más caro mientras los estándares de derechos humanos son implementados en las instalaciones de custodia.

Esta plataforma de acción no brindará por sí misma de derechos humanos a las prisiones, pero si facilitará su inserción y defensa. Más importante aún, disminuirá la ilegalidad incorporada y reducirá la corrosividad humana dentro de la prisión. Por último, debemos preguntarnos por la dicotomía ofrecida por los derechos humanos tras las rejas y los derechos de los ciudadanos fuera de ellas. Tanto los presos como los ciudadanos debiesen tener derecho a un mínimo de capacidades sociales y dignidad, ya sea dentro o fuera de los centros penitenciaros.

\footnotetext{
${ }^{14}$ Esto lo ha demostrado TYLER, Tom R., Why People Obey the Law. Princeton: Princeton University Press, 2006.
} 
WACQUANT, Loïc "La futilidad y necesidad de los derechos humanos en la era de hiper inflación carcelaria".

\section{BIBLIOGRAFÍA}

BLACK, Timothy, When a Heart Turns Rock Solid: The Lives of Three Puerto Rican Brothers On and Off the Streets, New York: Vintage, 2010.

BOURDIEU, Pierre, "Rethinking the State: Genesis and Structure of the Bureaucratic Field", Sociological theory 12, no. 1, (1994), pp. 1-18.

BURKE, Kenneth, The Philosophy of Literary Form, Berkeley: University of California Press, 1957.

CARDENAS, Sonia, Human Rights in Latin America: A Politics of Terror and Hope, Philadelphia: University of Pennsylvania Press, 2011.

CLAPHAM, Andrew, Human Rights: A Very Short Introduction, Oxford: Oxford University Press, 2016.

COMFORT, Megan, Doing Time Together: Love and Family in the Shadow of the Prison, Chicago: University of Chicago Press, 2008.

DIRK, van Zyl Smit; SNACKEN, Sonja, Principles of European Prison Law and Policy: Penology and Human Rights, Oxford: Oxford University Press, 2009.

ERIKSON, Kai, Wayward Puritans: A Study in the Sociology of Deviance, New York: Wiley, 1996.

FOUCAULT, Michel, Discipline and Punish: The Birth of the Prison, Trad. SHERIDAN, Alan, New York: Vintage, new ed. 2012.

GARLAND, David, "Penal Modernism and Postmodernism", en: BLOMBERG, Thomas G.; COHEN, Stanley (Eds.), Punishment and Social Control. New York: Aldine de Gruyter, 2nd expanded edition, 2003.

GELTNER, Guy, The Medieval Prison: A Social History, Princeton: Princeton University Press, 2008.

GOFFMAN, Erving, Asylums: Essays on the Social Situation of Mental Patients and Other Inmates, New York: Anchor, 1961.

GUILHOT, Nicolas, The Democracy Makers: Human Rights and International Order, New York: Columbia University Press, 2005.

HATHAZY, Paul Carlos, Democratizing Leviathan: Bureaucrats, Experts and Politics in the Transformation of the Penal State in Argentina and Chile. Berkeley, unpublished Ph.D dissertation, pp. 1-332, 2013.

HATHAZY, Paul; MULLER, Markus-Michael, "The Rebirth of the Prison in Latin America: Determinants, Regimes and Social Effects", Crime, Law and Social Change 65, no. 3 (2016), pp. 113-135.

HOFFMAN, Kelly; CENTENO, Miguel Angel, "The Lopsided Continent: Inequality in Latin America", Annual Review of Sociology 29, (2003), pp. 363-390.

IRWIN, John, The Felon, Berkeley: University of California Press, 1987.

MÉNDEZ, Juan E.; O’DONNELL, Guillermo A.; PINHEIRO, Paulo Sérgio (Eds.), The (Un) Rule of Law and the Underprivileged in Latin America. South Bend, Indiana: University of Notre Dame Press, 1999.

MULLER, Markus-Michael, "The Rise of the Penal State in Latin America", Contemporary Justice Review 15, no. 1 (2012), pp- 57-76.

RUSCHE, Georg; KIRSCHEIMER, Otto, Punishment and Social Structure, New Brunswick: Transaction Books, 2003. 
Polít. crim. Vol. 12, No 24 (Diciembre 2017), Doc. 2, pp. 1264-1274.

[http://www.politicacriminal.cl/Vol_12/n_24/Vol12N24D2.pdf]

SANHUEZA, Guillermo; BRANDER, Francisca; FUENZALIDA, Fernando, "First Survey on Prison Life in Chile: A Social Work Call for Prison Reform." International Social Work: in press, 2017.

SIKKINK, Kathryn, Mixed Signals: US Human Rights Policy and Latin America, Ithaca: Cornell University Press, 2007.

SOMERS, Margaret R.; ROBERTS, Cristopher N.J., "Toward a New Sociology of Rights: A Genealogy of 'Buried Bodies' of Citizenship and Human Rights", Annual Review of Law and Social Science 4, (2008), pp. 385-425.

SPIERENBURG, Pieter, The Spectacle of Suffering: Executions and the Evolution of Repression, Cambridge: Cambridge University Press, 1984.

TYLER, Tom R., Why People Obey the Law. Princeton: Princeton University Press, 2006.

WACQUANT, Loïc, Punishing the Poor: The Neoliberal Government of Social Insecurity, Durham and London: Duke University Press, 2009.

, "The Wedding of Workfare and Prisonfare Revisited", Social Justice 38, 1-2 (Spring, 2011), pp. 203-221.

WAKEFIELD, Sara; WILDEMAN, Christopher, Children of the Prison Boom: Mass Incarceration and the Future of American Inequality. New York: Oxford University Press, 2013.

WEBER, Max, The Methodology of the Social Sciences, Glencoe: Free Press, 1948. 